\title{
Improved Belief Propagation by Employing the Positive Effects of the Feedback Messages
}

\author{
Cui Junyun*, Ma Bei \\ Chang'an District, Xi'an, Shaanxi, China. \\ * Corresponding author. Tel.: +86-029-81556317; email: 02044027@163.com \\ Manuscript submitted February 24, 2019; accepted April 16, 2019. \\ doi: $10.17706 /$ jcp.14.4.295-301
}

Department of Computer Science, Xi'an University of Finance and Economics, No. 360 of Chang Ning Road,

\begin{abstract}
In this paper, a modification to belief propagation (BP) decoding algorithm is proposed, which is based on extracting the prior messages of each variable node to help the BP decoding, and is particularly effective for low-density parity-check (LDPC) codes with short cycles, where the existence of cycles makes the original BP algorithm perform suboptimal. The proposed algorithm, referred to as "employing the positive effects of the feedback messages (EPEFM)", extracts the positive effects of the feedback messages and then makes use of them as prior messages to assist the decoding of the BP algorithm. Simulation results confirm the effectiveness of our proposed algorithm, which improves the performance in high signal-to-noise-ratio (SNR) region without loss in low SNR region.
\end{abstract}

Key words: Belief propagation (BP), cycle, Tanner graph, low-density parity-check (LDPC) codes.

\section{Introduction}

Gallager firstly proposed low-density parity-check (LDPC) codes, along with several message-passing decoding algorithms [1], among which the belief propagation (BP) algorithm is shown to have the best performance. It has been proved in [2], [3] that the BP algorithm on cycle-free Tanner graph converges to the optimal solution but requires large computational complexity. LDPC codes with large enough lengths decoded with BP decoding algorithm have capacity approaching performance [4], [5]. However, in many applications LDPC codes have short and moderate lengths. In this case, the BP decoding algorithm loses its optimality because the inevitable small cycles create the dependencies when messages pass through cycles.

On one hand, a great number of methods to construct large girth (the length of the shortest cycle) LDPC codes with short and moderate lengths under BP decoding algorithm are developed such as in [6], [7], which are based on the observation that the error rate decreases exponentially with the number of independent iterations. On the other hand, a few modified versions of the BP algorithm that outperform the standard BP algorithm have been introduced in [8]-[13]. In particular, an improvement method by properly scaling down the variable to check extrinsic logarithm likelihood ratio (ex-LLR) using a (multiplicative versus additive) factor ( $\alpha$ versus $\beta$ ) in the iterative process of BP algorithm has been reported [8], [9]. Another method [10] attenuates the check to variable ex-LLR, when sudden sign change happens in successive decoding iterations. However, it is still interesting to find a theoretical way to determine appropriate attenuation factors. In [11]-[13], several scheduling algorithms, including probabilistic versus deterministic schedule and node based versus edge based schedule, are presented.

In this letter, a modification to BP algorithm is proposed, which provides non-negligible improvement 
over the performance of standard BP algorithm, particularly with short cycle lengths.

\section{LLR-BP Algorithm}

A binary LDPC code $C$ is described by a sparse parity-check matrix $H=\left[\mathrm{H}_{i j}\right]$. Let us denote the set of variable nodes that participate in check node $\mathrm{C}_{i}$ by $N_{i}=\left\{j: \mathrm{H}_{i j}=1\right\}$, and the set of check nodes in which variable node $\mathrm{V}_{j}$ participates by $M_{j}=\left\{i: \mathrm{H}_{i j}=1\right\}$. We also denote a set $N_{i}$ with variable node $\mathrm{V}_{j}$ excluded by $N_{i} \backslash j$, and a set $M_{j}$ with check node $\mathrm{C}_{i}$ excluded by $M_{j} \backslash i$. In the following, we assume binary phase shift keying (BPSK) modulation, which maps a codeword $c=\left(c_{0}, c_{1}, \cdots, c_{N-1}\right) \in C$ into a modulated sequence $x=\left(x_{0}, x_{1}, \cdots, x_{N-1}\right)$ according to $\mathrm{x}_{j}=1-2 \mathrm{c}_{j}$ for $j=0,1, \cdots, \mathrm{N}-1$. The sequence $x$ is then transmitted over the additive white Gaussian noise (AWGN) channel. The received sequence $y=\left(\mathrm{y}_{0}, \mathrm{y}_{1}, \cdots, \mathrm{y}_{N-1}\right)$ is given by

$$
y_{j}=x_{j}+n_{j}, \quad j=0,1, \cdots, N-1
$$

where $\mathrm{n}_{j}$ are independent and identically distributed Gaussian random variables with zero mean and variance $N_{0} / 2$.

Let $\mathrm{r}_{j}$ denote the soft LLR output of the AWGN channel and initially set $\mathrm{r}_{j}=\left(4 / N_{0}\right) \mathrm{y}_{j}$. Let $\mathrm{L}_{i j}^{(l)}$ and $\mathrm{z}_{i j}^{(l)}$ be the LLRs sent from check node $\mathrm{C}_{i}$ to variable node $\mathrm{V}_{j}$, and from the variable node $\mathrm{V}_{j}$ to check node $\mathrm{C}_{i}$ at the $l$-th iteration of $\mathrm{BP}$ decoding, respectively. Let $\mathrm{z}_{j}$ denote the posteriori LLR of variable node $\mathrm{V}_{j}$. For the completeness, the LLR-BP algorithm is carried out as follows.

Initialization: Set $l=1$, and the maximum number of iterations to $\mathrm{I}_{\max }$. For each $i, j, \mathrm{~L}_{i j}^{(0)}=0$.

Step 1: (i) Vertical step: for $0 \leq j \leq N-1$ and each $i \in M_{j}$, process:

$$
\begin{gathered}
\mathrm{z}_{i j}^{(l)}=\mathrm{r}_{j}+\sum_{i^{\prime} \in \mathcal{M}_{j} \backslash i} \mathrm{~L}_{i^{\prime} j}^{(l-l)} \\
\mathrm{z}_{j}^{(l)}=\mathrm{r}_{j}+\sum_{i^{\prime} \in \mathcal{M}_{j}} \mathrm{~L}_{i^{\prime} j}^{(l-l)}
\end{gathered}
$$

(ii) Horizontal step: for $0 \leq j \leq N-1$ and each $i \in \mathcal{M}_{j}$, process:

$$
\mathrm{L}_{i j}^{(l)}=2 \tanh ^{-1}\left(\prod_{j^{\prime} \in \mathcal{M}_{i} \backslash j} \tanh \left(\frac{\mathrm{z}_{i j^{\prime}}^{(l)}}{2}\right)\right)
$$

Step 2: Hard decision and stopping criterion test:

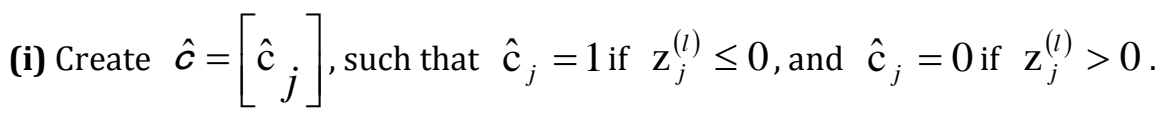

(ii) If $\hat{c} H^{T}=0$ then stop the decoding iteration and $\hat{c}$ is considered as a valid decoding result. Otherwise, set $l=l+1$ and go to Step 1. A failure is declared if the maximum iteration number $\mathrm{I}_{\max }$ is reached without a valid decoding. 


\section{Improved LLR-BP Algorithm}

We now proceed to discuss the sub-optimality of the standard BP algorithm. Consider a graph with cycles, throughout the iterative decoding process, dependency is created between the incoming messages to a variable node and its soft LLR output of the channel or among the incoming messages to a variable node.

In these cases, several scheduling algorithms [11]-[13] are proposed, which improve the performance of the standard BP algorithm by updating the outgoing messages of a node (an edge) with an average frequency proportional to the length of local girth and the length of the shortest closed path passing that node (edge). However, to implement these schedules, we found that although the cross-dependency between the incoming messages to a variable node and its initial message is eliminated, the independency between the incoming messages to a variable node and its initial message is also eliminated. For example, in Fig. 1, the incoming message to variable node $V_{l}$ is derived not only from the initial message of $V_{l}$ due to short cycles but also from the initial messages contributed by other variable node $\mathrm{V}_{j},(0 \leq j \leq N-1, j \neq 1)$.

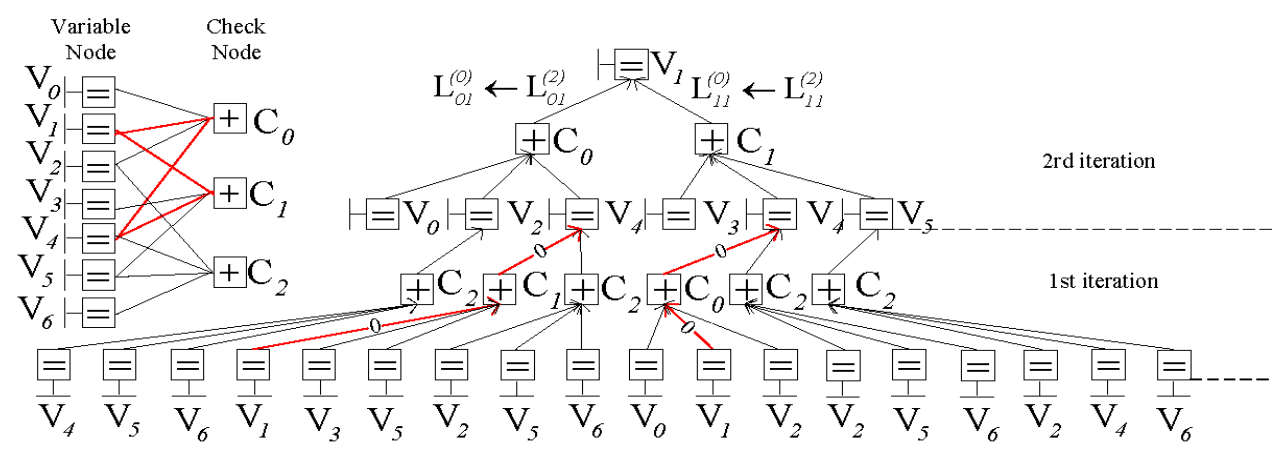

Fig. 1. A normal graph and an iterative computation of prior messages for the node $\mathrm{V}_{l}$ by the proposed algorithm with $l_{\max }=4$ in its subgraph.

Based on the consideration above, we extract the positive effects of the feedback messages of each variable node, throughout the iteration decoding process, to replace the prior message in the standard BP algorithm. This extraction can eliminate the dependencies between the feedback messages of each variable node and its initial message through some closed paths and preserve the positive effects of the feedback messages from the other nodes.

Instead of predetermining the maximal possible length of the closed paths passing through each variable node $\mathrm{V}_{j}$, we consider the paths only up to a given maximal length $l_{\max }$ for that variable node, i.e. $l_{\max } / 2$ is the number of iterations for extracting the prior messages of $\mathrm{V}_{j}$.

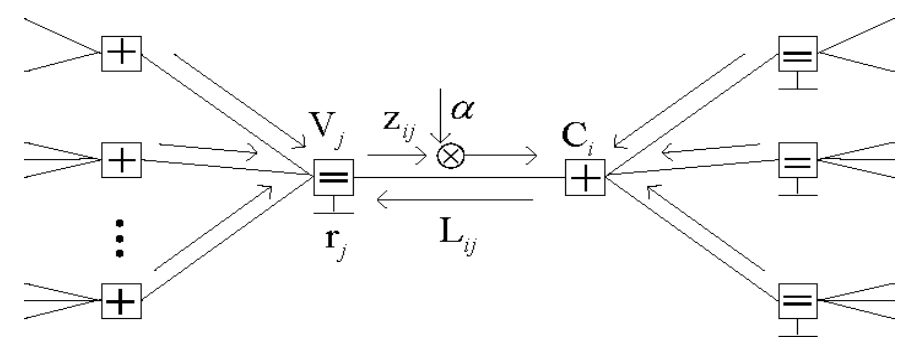

Fig. 2. Normal graph of the proposed node update rules. When the prior message $\mathrm{L}_{i j}^{(0)}$ is iteratively computed, $\alpha=0$ for $\mathrm{V}_{j^{\prime}}, j=j^{\prime}$; otherwise, $\alpha=1$. 
The only difference between the proposed BP algorithm and the LLR-BP algorithm lies in the value of the prior messages in the initialization. As shown in Fig. 2, the proposed BP algorithm can be reduced to the LLR-BP algorithm, when $\alpha=1$ is chosen. Prior messages of the proposed BP algorithm are obtained iteratively as follows:

Extraction: Stage 1: Set $l=1$, and the initial number of iterations to $l_{\max } / 2$.

Stage 2: For $0 \leq j \leq N-1$ and each $i \in M_{j}$, process jointly the following four steps:

(i) For each $i^{\prime \prime}, j^{\prime \prime}, \quad \mathbf{z}_{i^{\prime \prime} j^{\prime \prime}}^{(0)}=\mathrm{r}_{j^{\prime \prime}}$.

(ii) Horizontal step: for each $i \in M_{j}$, set

$$
\mathbf{z}_{i j}^{(l-1)}=\alpha \times \mathbf{z}_{i j}^{(l-1)}=0
$$

For $0 \leq j^{\prime \prime} \leq N-1$ and each $i^{\prime \prime} \in M\left(j^{\prime \prime}\right)$, process with (4).

(iii) Vertical step: for $0 \leq j^{\prime \prime} \leq N-1$ and each $i^{\prime \prime} \in M\left(j^{\prime \prime}\right)$, process with (2).

(iv) If the initial iteration number $l_{\max } / 2$ is reached, for each $i \in M_{j}$, restore $\mathrm{L}_{i j}^{\left(l_{\max } / 2\right)}$ and let $l=1$. Otherwise, set $l=l+1$ and go to (ii).

Initialization: For each $i, j, \quad \mathrm{~L}_{i j}^{(0)}=\mathrm{L}_{i j}^{\left(l_{\max } / 2\right)}$. Set the maximum number of iterations to $\mathrm{I}_{\max }$.

For example, in Fig. 1 the prior messages of the node $V_{1}$ are extracted by the proposed modification with $l_{\max }=4$. It can be seen that $\mathrm{L}_{01}^{(2)}$ and $\mathrm{L}_{l 1}^{(2)}$ are the messages dependent on the outgoing messages from the variable node sets $\left\{\mathrm{V}_{0}, \mathrm{~V}_{2}, \mathrm{~V}_{4}, \mathrm{~V}_{5} \mathrm{~V}_{6}\right\}$ and $\left\{\mathrm{V}_{2}, \mathrm{~V}_{3}, \mathrm{~V}_{4}, \mathrm{~V}_{5} \mathrm{~V}_{6}\right\}$, respectively. The resulting prior messages for variable node $V_{1}$ make use of the positive effects of its feedback messages.

Since the positive effects of the feedback messages of all the variable nodes are considered in the initial process, this proposed algorithm can achieve decoding performance superior to the BP algorithm.

\section{Simulation Results}

To investigate the performance of proposed BP algorithm, we give the simulation results on two LDPC codes. Both codes are described by their parity-check matrices and constructed by computer random search. Code I is a rate $1 / 4,(200,50)$ LDPC code with row and column weights 4 and 3 , respectively. Code II is a rate $1 / 2,(504,252)$ LDPC code with row and column weights 6 and 3 , respectively. Their average girths are 6.62 and 6.19, respectively. For both codes, the maximum number of iterations is 60 . For all the simulations, enough codewords are simulated to generate at least 100 codeword errors for each $E_{b} / N_{0}$.

Fig. 3 and Fig. 4 show the block error rate (BLER) and bit error rate (BER) curves for code I and II with different decoding algorithms, respectively. It can be seen that, for both block lengths, at high SNR values, the EPEFM algorithm outperforms the standard BP algorithm, the algorithm of [9] and the edge-based probabilistic scheduling decoding [12] considerably. At lower SNR values, the proposed algorithm never performs worse than the standard BP algorithm.

Fig. 3 depicts the average number of iterations of the EPEFM with $l_{\max }=10$ (excluding the iteration of extracting the positive effects of the feedback messages for each variable node), edge-based probabilistic schedule decoding [12], algorithm of [9] with $\alpha=0.65$ and standard BP algorithms. 


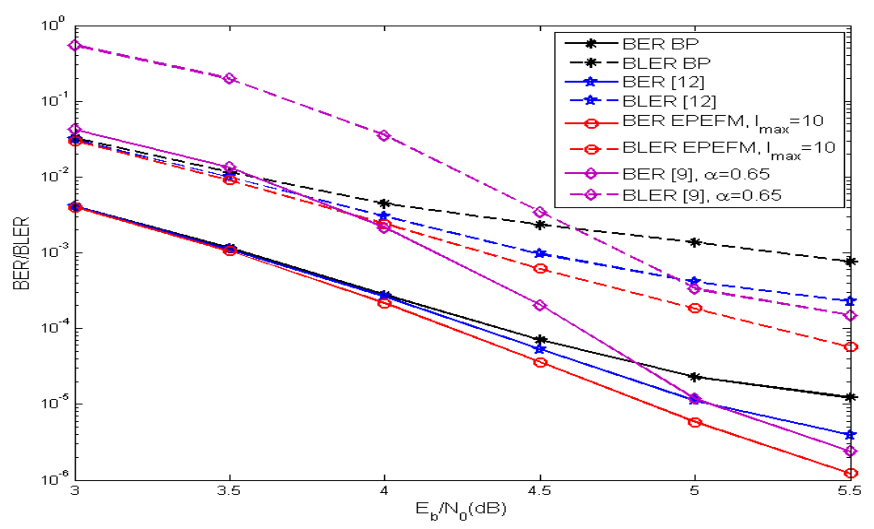

Fig. 3. Error performance for iterative decoding of code I.

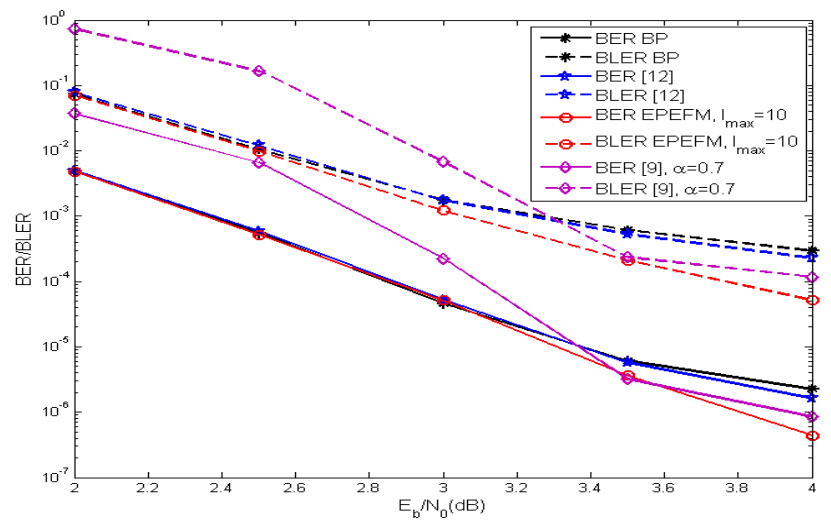

Fig. 4. Error performance for iterative decoding of code II.

It can be seen that the EPEFM algorithm converges faster than the other two algorithms. But note that the complexity of the process of extracting the extrinsic messages of each variable node without any simplification is a monotonically increasing function of the block-length and the target maximal length $l_{\max }$ of the closed paths.

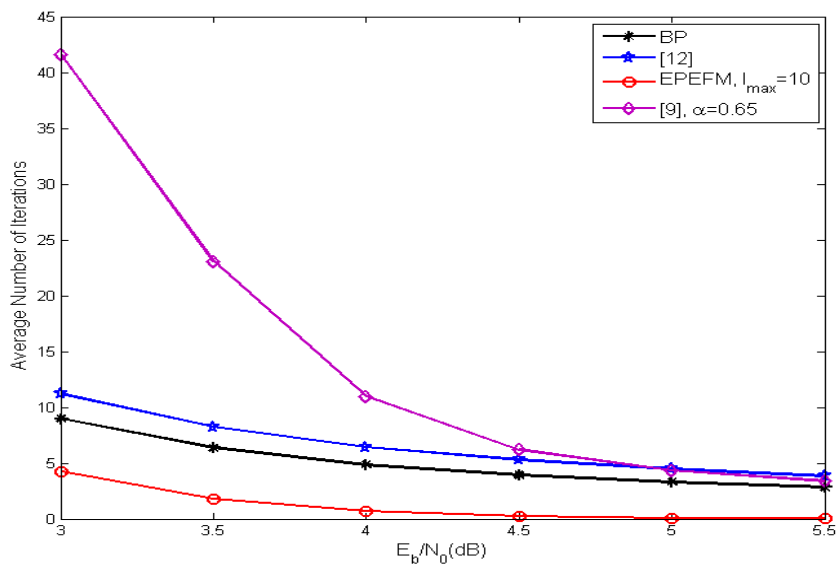

Fig. 5. Average number of iterations for iterative decoding of code I.

\section{Conclusion}

Building upon the sub-optimality of the probabilistic scheduling decoding algorithms, we propose a new 
modified BP algorithm referred to as "employing the positive effects of the feedback messages (EPEFM)" algorithm. This algorithm is based on extracting the positive effects due to short cycles in the associated Tanner graph instead of stopping updating the messages passing through the edges in the found cycles. We have showed by simulation that the EPEFM algorithm, at the cost of complexity in the process of extracting the positive effects of the feedback messages for each variable node, improves substantially upon the overall BER and BLER. For a given Tanner graph of an LDPC code, particularly with short cycles, there appears to be a great potential for performance improvement, through employing the positive effects due to the feedback messages. The results of this work confirm that for pushing the performance of iterative BP algorithms much closer to their limit, an appropriate use of the positive effects of the feedback messages is a necessity.

\section{Acknowledgment}

This paper is supported by the Project of Teaching and Research in Xi'an University of finance and economics (18FCJH02), and the Project of National Statistical Science Research (2018LY55).

\section{References}

[1] Gallager, R. G. (1962) . Low density parity check codes. IEEE Trans. Inf. Theory, 8(1), 21-28.

[2] Tanner, R. M. (1981). A recursive approach to low complexity codes. IEEE Trans. Inf. Theory, 27(5), 533-547.

[3] Wiberg, N., Loeliger, H. A., \& Kotter, R. (1995). Codes and iterative decoding on general graphs. Euro. Trans. Telecommun., 6(5), 513-525.

[4] MacKay, D. J. C., \& Neal, R. M. (1996). Near Shannon limit performance of low density parity check codes. IEE Electron. Lett., 32(18), 1645-1646.

[5] Saeedi, H., Pishro-Nik, H., \& Banihashemi, A. H. (2011). Successive maximization for the systematic design of universally capacity approaching rate-compatible sequences of LDPC code ensembles over binary-input output-symmetric memoryless channels. IEEE Trans. Commun., 59(7), 1807-1819.

[6] Tao, X., Li, Y., Liu, Y., \& Hu, Z. (2018). Constructing Large Girth Qc Protograph Ldpc Codes Based On Psd-Peg Algorithm. IEEE Commun. Lett., 22(1), 9-12.

[7] Jiang, X., Hai, H., Wang, H. Lee, M. H. (2017). Constructing large girth QC protograph LDPC codes based on PSD-PEG algorithm. IEEE Access, 5, 13489-13500.

[8] Sun, R., Hou, X., Sun, J., Guan, H., \& Zhao, Z. (2018). Reliability-based-layered belief propagation for iterative decoding of LDPC codes. Proceedings of IEEE International Symp. on Information Theory.

[9] Li, P., De Lamare, R. C., \& Liu, J. (2018). Knowledge-aided iterative detection and decoding for multiuser multiple-antenna systems. IET Commun., 12(11), 1373-1379.

[10] Al-Rubaye, G. A., Tsimenidis, C. C., Johnston, M. (2017). Low-density parity check coded orthogonal frequency division multiplexing for PLC in non-Gaussian noise using LLRs derived from effective noise probability density functions. IET Commun., 11(16), 2425-2432.

[11] Jiao, X., Mu, J., \& Wei, H. (2017). Reduced complexity node-wise scheduling of ADMM decoding for LDPC codes. IEEE Commun. Lett., 21(3), 472-475.

[12] Aslam, C. A., Guan, Y., Cai, K., \& Han, G. (2017). low-complexity belief-propagation decoding via dynamic silent-variable-node-free scheduling. IEEE Commun. Lett., 21(1), 28-31.

[13] Aslam, C. A., Guan, Y., \& Cai, K. (2017). edge-based dynamic scheduling for belief-propagation decoding of LDPC and RS codes. IEEE Trans. Commun., 65(2), 525-535. 


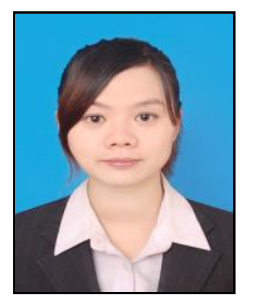

Cui Junyun was born in China in 1984. She received the Ph.D degree in tele-communication engineering from Xidian University, China in 2012. She is currently an associate professor at Xi'an University of Finance and Economics. Her research interests include channel encoding, channel decoding and neural network algorithm.

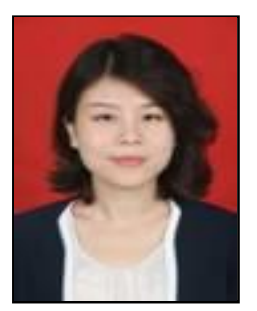

Ma Bei was born in China in 1982. She received the Ph.D degree in tele-communication engineering from Xidian University, China in 2016. She is currently an associate professor at Xi'an University of Finance and Economics. Her research interests include wireless communication. 